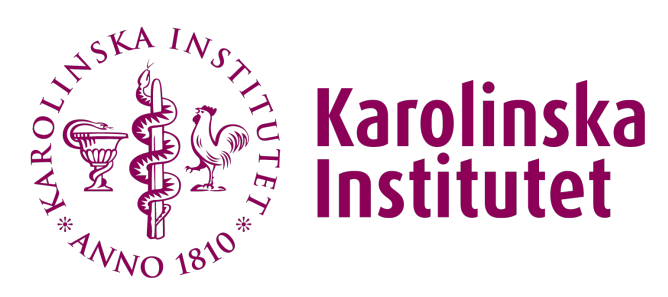

Karolinska Institutet

http://openarchive.ki.se

This is a Peer Reviewed Accepted version of the following article, accepted for publication in Technology and Disability.

\title{
Factors that impact the level of difficulty of everyday technology in a sample of older adults with and without cognitive impairment
}

Technol Disabil. 2011 Jan;23(4):243-50.

Andover Medical Publishers

http://doi.org/10.3233/TAD-2011-0331

http://hdl.handle.net/10616/40956

If not otherwise stated by the Publisher's Terms and conditions, the manuscript is deposited under the terms of the Creative Commons Attribution-NonCommercial-NoDerivatives License (http://creativecommons.org/licenses/by-nc-nd/4.0/), which permits non-commercial re-use, distribution, and reproduction in any medium, provided the original work is properly cited, and is not altered, transformed, or built upon in any way. 


\section{(19) \\ Karolinska Institutet}

This is an author produced version of a paper published in Technology and Disability. This paper has been peerreviewed but does not include the final publisher proofcorrections or journal pagination.

Citation for the published paper:

\section{Ann-Helen Patomella, Anders Kottorp, Camilla} Malinowsky, Louise Nygård. Factors that impact the level of difficulty of everyday technology in a sample of older adults with and without cognitive impairment. Technology and Disability. 2011;23(4):243-250.

URL: http: / /dx.doi.org/10.3233/TAD-2011-0331

Access to the published version may require subscription. Published with permission from: IOS Press 
Title: Factors that impact the level of difficulty of everyday technology in a sample of older adults with and without cognitive impairment

Ann-Helen Patomella, PhD, Occupational Therapist, Department of Neurobiology, Care

Sciences and Society, Division of Occupational Therapy, Karolinska Institutet

Anders Kottorp, PhD, Occupational Therapist, Department of Neurobiology, Care Sciences and Society, Division of Occupational Therapy, Karolinska Institutet

Camilla Malinowsky, PhD Student, Occupational Therapist, Department of Neurobiology,

Care Sciences and Society, Division of Occupational Therapy, Karolinska Institutet

Louise Nygård, PhD, Professor of Occupational Therapy, Department of Neurobiology, Care

Sciences and Society, Division of Occupational Therapy, Karolinska Institutet

\section{Corresponding Author:}

Louise Nygård, Division of Occupational Therapy, Department of Neurobiology, Care

Sciences and Society, Karolinska Institutet,

Phone number +46852483792

e-mail louise.nygard@ki.se

Post address: Department of Neurobiology, Care Sciences and Society, 23200, Division of

Occupational Therapy, Karolinska Institutet, 14183 Huddinge 


\begin{abstract}
Everyday technologies (ET) are an important part of the environment in which we live and interact, and older adults with cognitive impairments have to be able to manage ETs in order to participate in society. The aim of the present study was to bring new insight into what makes an ET easy or difficult to use for older adults with and without cognitive impairment. Older adults with or without cognitive impairments $(n=116)$ were observed managing 27 ETs. In order to analyze and detect variables that influenced the level of difficulty of the ETs managed, a regression analysis was used and predefined assumptions were investigated. The results revealed that ETs that were used less than once a week were more difficult to handle, as were those with a complex design. The results suggests that ETs, more specifically Information and Communication Technology, need to be designed to be more user-friendly and less complex, and older adults that wish to continue using an ET need to be frequent users. The age and gender of the user, and how long the ET had been in use did not relate to how difficult an ET was to manage.
\end{abstract}

Keywords: Mild Cognitive Impairment, Alzheimer's Disease, Older Adults, Occupational Therapy, Disability 


\section{Introduction}

Everyday technology (ET) is an important part of the environment in which we live and interact [1]. We need to use technology for everyday life activities such as calling a friend or contacting the authorities, or for just cooking a simple dinner on the stove. Older adults with cognitive impairments who still live at home have to be able to manage everyday technologies like everybody else in order to participate in society. Everyday technology could have the potential to facilitate the daily lives of older people. However, we know little of what makes everyday technology difficult to use, and how older adults with cognitive impairment manage technology, and how this may interfere with their potential independence, wellbeing and participation in society $[2,3]$. In this study, the concept of everyday technology is used to incorporate electronic, technical, and mechanical artifacts, and systems that are generally used by Swedish older adults with and without cognitive impairment in the study context.

Studies show that older adults living at home who have cognitive impairments such as mildstage dementia or Mild Cognitive Impairment (MCI) experience difficulties in using ET [3, 4]. Nygård and Starkhammar [3] showed from extensive interview material that people with dementia experienced difficulties using ET; for example in identifying, interpreting and knowing how to respond to the information received from the ET, in using and selecting a command or button from a variety of alternatives, or handle two pieces of an ET when for example inserting a cassette into a player. Additionally, older adults with dementia and MCI reported fewer ETs to be relevant for their everyday living as compared to those without cognitive impairments [4]. A recent study also indicated that on a group level, people with mild-stage Alzheimer's Disease (AD) or mild cognitive impairment (MCI) had a decreased 
ability to manage ETs compared to older adults without cognitive impairment, using the Management of Everyday Technology Assessment (META) [5]. META has been developed to measure the ability to manage ET in everyday life for older adults in general, and more specifically for people with mild-stage AD or MCI. A study using a Rasch measurement model (Linacre, 2006) showed that the psychometric properties of META were promising, indicating acceptable scale and person response validity [6]. In order to facilitate the participation of people with cognitive impairments in everyday life, it is important to increase our knowledge not only of the difficulties they may face when using ET, but also of what makes an ET easy or difficult to use. The Rasch analysis rank orders the ETs within META in a hierarchy from the most difficult to the easiest for the users [6, 7], but the Rasch analysis does not tell us why the ETs are more or less difficult. In the present study we made four assumptions about what makes an ET difficult to manage for older adults with and without known cognitive impairments, based on earlier research findings and contemporary discussions in the research literature.

1) We assumed that more recently developed and computerized ETs would be more difficult to use for older adults with or without cognitive impairment than ETs used for domestic activities. Studies have pointed out difficulties among people with dementia in using more recently developed ETs; for example one study showed the difficulties people with dementia had in managing even basic telephone tasks for example knowing how to use the dialing code [8], and another presented their difficulties in handling mobile phones and computers [3]. In a recent study, people with and without cognitive impairment were interviewed about how they perceived the difficulty of different ETs at home and in society, and the results showed that computers and mobile phone technologies were perceived as the most difficult to use [9]. Due to these earlier findings and a more general received wisdom, we assumed that ETs that were 
computerized would be more difficult to use than the more traditional ETs used in domestic activities.

2) We assumed that ETs with a more complex design would be more difficult to use. Further, we assumed that more difficult ETs would require the user to carry out more actions (as measured in META) than easier ETs. Lewis, Langdon and Clarkson [10] found that a more complex design could affect performance, especially among the elderly; they showed that there was a significant difference in difficulty for older adults to operate two differently designed microwave cookers. The microwave cooker with a dial interface was easier to learn to use than the cooker with a button interface. Lewis and colleagues presumed that the reason for this was that the button interface requires a higher number of alternative control action decisions, and less tactile and visual feedback on the results of actions is given than is the case with the dial interface. In this study the META was used to measure the individuals' ability to manage ETs when using up to ten skill actions, and the frequency of required skills depends on how many skills are needed in order to be able to manage a specific ET[6]. The META also includes an observation of whether the design of the ETs facilitates or hinders the individual's performance. We assumed that a more complex design could make the management of an ET more difficult to use and would require more skills.

3) Our third assumption was that the estimated difficulty of the ET would be influenced by the age of the users, i.e. more challenging ETs would be managed by younger users and easier ETs would be managed by older users. Older adults have been found to have more difficulties handling ETs than younger people [5, 10]. In earlier studies $[2,11,12]$ older age has been found to correlate with non-use of Information and Communication Technology (ICT), especially computers. Selwyn and colleagues [12] conducted a survey in 2002 and found that older adults using computers were more likely to be male and under the age of 70 years. The reasons for non-use of computers were explained as the technology's irrelevance to the 
everyday lives of the elderly (25\%), the perception of being too old (21\%), while inability was mentioned by $13 \%$. Similarly, Larsson [13] found that the oldest old (over the age of 85 years) experienced difficulties in using ET and that they needed to experience the ETs as beneficial in their everyday lives to be incorporated and used. While the present study aimed to investigate the difficulty in managing ETs rather than use and non-use, we still assumed that age would influence the ET's level of difficulty.

4) Our fourth assumption was that the difficulty of an ET would be influenced by the habits of the user, and that ETs that had been used often and for a long time would be easier to manage than more recently incorporated and less frequently used ETs. There is a lack of studies related to habits and use of ETs, but Nygård [14] found in a qualitative study that frequent and daily use was important for a continued use of ET in older adults with mild-stage dementia.

To summarize, earlier studies have indicated that design, age, and habits may influence an individual's use and management of an ET. In the present study we set out to test these predefined assumptions in order to determine what factors make an ET difficult to use for older adults. To our knowledge no study so far has empirically investigated existing ETs indepth to determine variables that contribute to their level of challenge, particularly when these variables interact with the characteristics of particular users. The aim of the present study was to bring new insight into what makes an ET easy or difficult to use for older adults with and without known cognitive impairment.The following research question was addressed: Which independent variables can explain the variation in the difficulty hierarchy of everyday technologies created from META?

\section{Methods}

\subsection{Subjects}


The present study is based on a sample of 116 older adults (Mean age: 73, Range 55-92, SD: 9,2) with and without cognitive impairment, and who were living at home. The inclusion criteria for all participants were: a) aged 55 years or older; b) that the participants should be active users of ET and motivated to participate in the study; c) that visual and hearing impairment should be compensated with appropriate devices; d) that participants with mildstage AD should have a Mini-Mental State-Evaluation (MMSE) [15] score of at least 17 from a maximum 30, participants with MCI should have a score of at least 24, and older adults with no known cognitive impairment should have a score of at least 27 . These cut-offs are often used to distinguish levels of cognitive function in screening for dementia. The participants with MCI and AD were recruited from investigation units for memory-deficits and day-care centres for people with dementia, while participants without cognitive impairment were recruited from the Society of Retirees and similar networks in the Stockholm area. The recruitment process has been further described by Malinowsky and colleagues [6]. Finally, of the 116 participants, 38 presented with mild-stage AD, 33 with MCI, and 45 older adults were without cognitive impairments. The detailed characteristics of the participants are described in Table 1. Ethical approval was obtained from one of the Regional Ethical Review Boards in Stockholm.

-Insert table 1 about here-

\subsection{Procedure}

The Management of Everyday Technology Assessment (META) was used to assess the ability to manage everyday technology of the participants. META consists of 10 skill items assessing observable performance actions, for example to identify and separate objects, to turn a knob or button in the correct direction, and to perform actions in a logical sequence (for a complete presentation of the 10 skill items, see [5]). Trained raters (occupational therapists) 
observed and scored the skill items using a three-category rating scale based on the difficulty of managing each ET; $3=$ no difficulty, $2=$ minor difficulty and $1=$ major difficulty (the scoring of the skill items is further described in the detailed manual by Nygård [16] and by Malinowsky [6]).

The data collection started with an open conversation using a set of pre-defined questions about the participant's everyday life related to ET, and resulted in a selection of ETs to be observed and assessed using META. Each participant was observed and scored on the management of two or more everyday technology situations, either in their own home or the neighbourhood. The ETs assessed were chosen by the participants and belonged to them, and thus were relevant to each person. They included cell phones, TVs, and computers. Questions were used to collect information related to habits and routines for using each ET (how often and for how long the participant had been using the ET) and how important the ET was for the participant. ETs used on a daily or weekly basis were categorized as used often; ETs used on a monthly, yearly, or even less seldom basis were categorized as used seldom. How long the ET had been used was recorded in units of months. To investigate the overall level of cognitive decline, the Mini-Mental Status Examination (MMSE) was used. The rater also observed and documented how the context and design affected the participant's use of the ET. The procedure for data collection has been further described by Malinowsky and colleagues $[5,6]$.

\subsection{Preliminary data analysis}

For the purposes of this study, i.e. in order to detect what makes an ET easy or difficult to use, the data analysis had to be divided into two steps; a preliminary step to prepare the data and a primary step consisting of a regression analysis. The preliminary analysis had two steps, as presented below; 


\subsubsection{Rasch analysis and item reduction}

The ordinal data from the all participants' observed management of ET as measured using META were transformed into equal interval measures using Rasch analysis [17]). In the Rasch analysis every ET received a measure of difficulty presented in log-odds probability unit (logits) on an interval scale representing the measured construct, i.e. management of everyday technology. FACETS computer software [17] rank-ordered the ETs $(n=68)$ into a hierarchy from easier to more difficult to handle. The participants chose by themselves what ETs they wanted to manage and this resulted in a wide spread of ETs that were observed and scored. For the purpose of this analysis, we pragmatically decided to exclude ETs that were observed and used by fewer than four participants since the estimation of the level of difficulty of these ETs would be associated with a large error of the estimate. As this criteria still could increase the risk of including ETs that did demonstrate too much unexpected patterns in the limited number of responses, as the numbers of evaluations per item could be considered too low $[18,19]$, we also monitored that the included technologies did demonstrate acceptable goodness-of-fit values. Acceptable goodness-of-fit was indicated by an infit $M n S q<1.5$ [7] associated with $z<2$ [20]. This reduction resulted in a remaining dataset of 27 ETs. All of these included technologies met the criteria for acceptable goodness-of-fit. Furthermore, as three ETs had been used only by either men or women we also excluded them from the data set since gender was an independent variable used in the subsequent regression analysis. These three excluded ETs were: sewing machine (only used by four women), shaver (only used by four men), and checking missed calls on a cell phone (only used by four men). After the final reduction, 24 ETs remained for the primary analysis. The remaining ETs were ordered in a hierarchy and categorized as an ET used either for ICT or for domestic activities.

\subsubsection{Converting variables representing personal characteristics into variables representing}

\section{ET characteristics}


The data set analyzed in the present study was based on variables that represented the characteristics of each participant in their use of a specific ET (originally used in Malinowsky and colleagues $[5,6])$. These variables had to be converted to represent the dependent variables i.e. the characteristics of each ET $(n=24)$. The conversion resulted in seven variables; Mean age of users, Gender proportion, Importance of the ET for the users, Impact of design on the use, Context for ET use, How often and For how long (it had been used). For example, the variable mean age was calculated from the age of the participants using the ET, i.e. the mean age of the users that were observed to use the electric stove was 76 years. For the variable proportion of gender, the proportion of male users of each ET was calculated.

After the second step in the preliminary analysis the data comprised seven variables that represented the characteristics of the ET and that we presumed could affect the level of difficulty of ETs when used by older adults, and in particular people with mild-stage AD or MCI. However, three of the seven variables had to be excluded from the primary analysis after more in-depth inspection and analysis of the data. We assumed that the perceived importance of the ET could affect the difficulty of the ET. However, the variable Importance of the ET for the user had to be excluded because there was a risk of an invalid relationship to the dependent variable (the ET's level of difficulty) since all participants had been instructed to demonstrate their use of ETs that were relevant and important for them. We assumed that the impact of the Design (of the ET) and Context (of where it was used) of the ET as well as How often and for How long the participants had been using the ET could affect the difficulty of the ET. However, Context (proportion of participants affected by the context in their management of ET, $\mathrm{r}=-0.25$ ) and How long (months of use, $\mathrm{r}=-0.16$ ) were not used in the primary analysis since simple correlation analyses showed a weak relationship to the dependent variable. In the end, four different variables were chosen for the primary analysis (see Table 2). 
Finally, in the preliminary analysis, a simple ranking of frequencies of used skill items was carried out. We assumed there would be a difference in the number of skills needed to handle the different ETs; i.e. that managing more difficult ETs would require more skill items than was the case for handling easier ETs. As previously presented, the META assesses ten observable skill items or actions that can be used when handling ETs, and only those that are observed to be used by the participant are scored. Descriptive statistics were used to investigate the mean number of skill items and range of items used per ET, and to correlate these with the item calibration measures of the ETs.

-Insert Table 2 about here-

\subsection{Primary data analysis}

Analysis of the data was carried out using IBM SPSS Statistics (version 18.0, IBM SPSS, Chicago, IL, 2010). A general linear model using a backward stepwise ANCOVA (Analysis of Covariance) was applied to identify which of the selected four variables could explain the variation in measures of everyday technology difficulty using META (dependent variable). The least significant available variable was eliminated at each step ( $p$-value for removal $>0.05$ ). The independent variables that were investigated were (a), Proportion of gender, (b) Mean age of users, (c) How often the ET was used, and (d) Impact of design on the use. Normal probability plots were visually inspected to ensure that the data were normally distributed. Correlations were used in order to investigate intra dependency between variables, and collinearity was investigated by ensuring Variance Influence Factors (VIF) of $\leq 10$. Adjusted R was used as a measure of the variance, explained by the independent variables.

\section{Results}

\section{1 Results from the preliminary analysis}


The Rasch analysis was based on all the participants' $(n=116)$ observed management of ET, and generated a hierarchy from the most difficult to the easiest ET to handle. A grouping of the items is presented in the item hierarchy, see Figure 1. ETs used for domestic activities seem to be generally easier than ICTs.

-Insert Figure 1 about here -

The mean frequency of skill items used for each ET revealed no difference across the ETs evaluated. The mean use of items was seven items per ET with a range of 5-8 items. Furthermore, there were no detectable differences in the range of skill items used within each ET. There was no significant relationship between the mean use of skill items and the ET difficulty measure $(r=0.19, p=0.3)$. The results indicated that there were no systematic differences in how many skill items were required to manage the 24 ETs included in the analysis.

\section{2 Results from the primary analysis}

The ANCOVA indicated that the variables How often $(\mathrm{F} 1,19=16.27)$ and Impact of design on the use $(\mathrm{F} 1,19=4.53)$ were significantly associated with the level of difficulty of the ETs, but that Mean age of users and Gender proportion were not (see table 3). The two variables of How often, and Impact of design on use in the final regression model could explain 51\% (adjusted R ) of the total variance in level of difficulty among the ETs. ETs that were used on a monthly, yearly, or less frequent basis were generally more difficult to manage than ETs used on a daily or weekly basis, and ETs with a more complex design were confirmed as being more difficult to use for the participants.

\section{-Insert Table 3-}

\section{Discussion}


The results from the present study both confirmed and contradicted our assumptions about what makes an ET difficult or easy to manage in people with or without cognitive impairment. The results show that the design and how often the person has been using the ET impact on how difficult an ET is to use. This is a confirmation of earlier research that has suggested that the design of the artifact $[9,10]$ and the habit of using it [14] are of importance for use or non-use of ETs for older adults with or without a cognitive impairment, provided that we also assume that ETs that are difficult to manage are likely not to be used.

Our first assumption for the study was that more recent and more computerized ETs would be more difficult to manage for older adults with or without cognitive impairment than more traditional or domestic ETs. As can be seen in Figure I, ICTs were more difficult than the domestic ETs, which supports our assumption.

Our second assumption was that ETs with a more complex design would be more difficult to manage, and this was confirmed in the regression analysis. Lewis and colleagues (2008) found that ETs with a more complex design were more challenging. These demanded that the user handle a greater number of alternative actions and gave less sensory feedback to the user When visually inspecting the most difficult ETs in the hierarchy (see Figure 1) we can see that they all have a design that is more complex (requiring the user to handle displays and other menus that are hidden in layers and are very complex) than for the easiest ETs, which have a design that is more visually self-instructing (e.g. stove or electric kettle) and requires less cognitive effort by the user.

Furthermore, we assumed that more difficult ETs would require the user to handle more actions (as measured in META) than easier ETs, but this was not confirmed since the preliminary analysis showed that there was no significant relationship between the ET difficulty measure and the mean frequency of skill items used per ET. This could be due to 
the fact that the aim and focus of the META is to observe and measure skills but it does not register how many times a skill is used. For example, from practice we know that the most difficult ET (TV: Video with remote control) requires the user to identify and discriminate objects several times since the user needs to identify both the TV and the video recorder as well as remote controls for both artifacts, while the easiest ET (electric stove) only requires the user to identify one artifact. However this process is not explicitly detected in META.

Our third assumption was that the difficulty of the ET would be influenced by the mean age of the users, i.e. that the ETs with a higher mean of older users would be less difficult.

Surprisingly the result of the present study did not confirm this assumption. Notably, earlier studies have focused on non-use and use of ETs and found that older adults (60 years and older) have more resistance towards using more challenging ETs like computers than younger users (21-60 years) [12]. Also, it has been found that younger people have a different view on and approach to ET, and use a wider variety of ETs than older adults [2, 13, 21]. The present study has a different focus, in analyzing age as a factor in the ability to manage ETs, rather than use and non-use of ICTs, and this makes it hazardous to compare the results with earlier research. Further research on age related to ability to manage ETs therefore is needed.

The fourth assumption was related to the habits of the user, and was that ETs that were used more often would be easier. The results confirmed that ETs that were used on a more frequent basis were easier to handle than those used less than once a week. Similar results about frequency of use of ETs have been found and discussed before [13, 14], but have not been empirically tested as a factor predicting the challenge using an ET. We also assumed that ETs that had been used for a longer time would be easier to handle than those more recently incorporated into use. However, in the early phase of the analysis, a simple correlation showed that the length of time a person had been using the ET did not impact on the difficulty of ETs. Larsson [13] found that very few older adults acquired new technology and they did 
not update older technology with new models. This suggests that a possible reason why How long ETs had been used did not correlate with the difficulty of the ET could be that the older adults in this study were less active consumers of new, updated technology, hence the ETs in the participants' homes and that were observed in this study had all been used for a long time, and so this variable was not relevant to the investigation.

In summary, the present study has shown that the more complex the design of an ET, the more difficult the ET will be to manage. Moreover, for this sample, ICTs are shown to be more difficult than domestic ETs, and ETs that are used more often are easier to manage than those used more seldom (less than once a week). This information can be used in clinical interventions, for example in guiding people with cognitive impairments to choose ETs with a less complex design and also to inform clients that if they need and wish to continue to use an ET they should be a frequent and habitual user. If older adults with cognitive impairments are provided with ETs that are less complex in design or guided in how to use them, their lives could become more independent and they might be able to participate in society to a greater extent. Together with earlier research findings, this study urges the technology market to provide ETs that are more user-friendly and less complex. 


\section{Acknowledgements}

The authors thank the participants who demonstrated their management of everyday technology. We also like to thank the occupational therapists who recruited and assessed the participants; Sofia Starkhammar, Monica Pantzar, Jenny Rasmussen Tjernlund, Susanne Andersson, Lizette Mårtensson and Maria Carlsson and the statistician Jakob Bergström at LIME, Karolinska Institutet. The research was funded by the Swedish Research Council, Swedish Brain Power, Botkyrka Community and The regional agreement on medical training and clinical research (ALF) between the Stockholm County Council and Karolinska Institutet. The American Alzheimer's Association also supported the study under the Everyday Technologies for Alzheimer's Care program. 


\section{References}

1. Emiliani, P.L., Assistive Technology (AT) versus Mainstream technology (MST): The research perspective. Technology and Disability, 2006. 18: p. 19-29.

2. Czaja, S., et al., Factors Predicting the Use of Technology: Findings From the Centre for Research and Education on Ageing and Technology Enhancement (CREATE). Psychology and Aging, 2006. 21(2): p. 333-352.

3. Nygård, L. and S. Starkhammar, The use of everyday technology by people with dementia living alone: Mapping put the difficulties. Aging \& Mental Health, 2007. 11(2): p. 144-155.

4. Rosenberg, L., L. Nygård, and A. Kottorp, Everyday Technology Usage (ETUQ) - evaluation of the psychometric properties of a new assessment of competence in technology use. Occupational Therapy Journal of Research, 2009. Online.

5. Malinowsky, C., et al., Ability to manage everyday technology: a comparison of persons with dementia or mild cognitive impairment and older adults without cognitive impairment. Assistive Technology, 2010. 5(6): p. 462-469.

6. Malinowsky, C., L. Nygård, and A. Kottorp, Psychmetric evaluation of a new assessment of the ability to manage technology in everyday life. Scandinavian Journal of Occupational Therapy, 2011. 18: p.26-35.

7. Bond, T. and C. Fox, Applying The Rasch Model: Fundamental Measurement in the Human Sciences. 2007, Mahwah, NJ: Lawrence Erlbaum Associates, Inc.

8. Nygård, L. and S. Starkhammar, Telephone use among non-institutionalized persons with dementia living alone: Mapping out difficulties and response strategies. Scandinavian Journal of Caring Sciences, 2003. 17: p. 239-249.

9. Rosenberg, L., Navigating Through Technological Landscapes: Views of people with dementia or $\mathrm{MCl}$ and their significant others. In Division of Occupational Therapy. 2009, Karolinska Institutet: Stockholm.

10. Lewis, T., P. Langdon, and P. Clarkson, Prior Experience of Domestic Microwave Cooker Interfaces: A User Study. In Designing Inclusive Futures., P. Langdon, J. Clarkson, and P. Robinson, Editors. 2008, Springer: London.

11. Selwyn, N., The information aged: A qualitative study of older adults' use of information and communications technology. Journal of Aging Studies, 2004. 18: p. 369-384.

12. Selwyn, N., et al., Older adults' use of information and communications technology in everyday life. Aging and Society, 2003. 23: p. 561-582.

13. Larsson, A. Everyday life amongst the oldest old-descriptions of doings and possesions and use of technology. In Department of Social and Welfare Studies. 2009, Linköpings Universitet: Norrköping.

14. Nygård, L., The meaning of everyday technology as experienced by people with dementia who live alone. Dementia, 2008. 7: p. 481-502.

15. Folstein, M.F., S.E. Folstein, and P.R. McHugh, "Mini-Mental State Examination": A practical method for grading the cognitive state of patients for the clinician. Journal of Psychiatric Research, 1975. 12: p. 189-198.

16. Nygård, L., Användarmanual för META. Svårigheter i teknologianvänding (research version of manual: Skills in using technology: an assessment). 2006, Stockholm: Division of Occupational Therapy, Karolinska Institutet.

17. Linacre, J., FACETS: Many-faceted Rasch measurement computer program. 1987-2006, MESA: Chicago.

18. Linacre, J., Sample size and item calibration stability. Rasch Measurement Transaction, 1994. 7 (4): p. 328 
19. Linacre, J., Optimizing rating scale category effectiveness. Journal of Applied Measurement,2002 3 (1): $p$ 85-106.

20. Wright, B., Linacre, J., Reasonable mean-square fit values. Rasch Measurement Transactions, 1994, 8(3): p.370

21. Olson, K.E., M.A. O'Brien, W.A. Rogers, and N. Charness. Diffusion of technology: Frequency of use for younger and older adults. Ageing International, 2011. 36: p. 123-145. 
Table 1: Characteristics of the participants

\begin{tabular}{|l|l|l|l|}
\hline Group & $\begin{array}{l}\text { Age (years), Mean } \\
(\mathrm{SD}), \text { range }\end{array}$ & $\begin{array}{l}\text { Gender, Male/Female } \\
(\% \text { male })\end{array}$ & $\begin{array}{l}\text { MMSE score, Mean } \\
(\mathrm{SD}), \text { range }\end{array}$ \\
\hline Older adults $(\mathrm{n}=45)$ & $73(10), 55-92$ & $16 / 29(36 \%)$ & $29(1), 27-30$ \\
\hline MCI $^{\mathrm{a}}(\mathrm{n}=33)$ & $70(8), 57-87$ & $19 / 14(58 \%)$ & $27(2), 24-30$ \\
\hline Mild-stage $\mathrm{AD}^{\mathrm{b}}(\mathrm{n}=38)$ & $75(9), 58-89$ & $18 / 20(47 \%)$ & $24(3), 17-29$ \\
\hline
\end{tabular}

${ }^{\mathrm{a}}=$ Mild Cognitive Impairment, ${ }^{\mathrm{b}}=$ Alzheimer Disease 
Table 2: Description of variables used in the regression model

\begin{tabular}{|l|l|}
\hline Variable & Description \\
\hline Gender proportion & The proportion (in \%) of male participants using the ET. \\
\hline Mean age of users & The mean age of the participants using the ET. \\
\hline How often & $\begin{array}{l}\text { The proportion (in \%) of participants using the ET seldom. Seldom } \\
\text { was defined as less than weekly use. }\end{array}$ \\
\hline $\begin{array}{l}\text { Impact of design on the } \\
\text { use }\end{array}$ & $\begin{array}{l}\text { The proportion (in \%) of participants affected negatively by the } \\
\text { design of the ET in their management. }\end{array}$ \\
\hline
\end{tabular}


Table 3: Results of backward stepwise ANCOVA on variables influencing the level of everyday technology difficulty (META hierarchy). Statistics given for the variables are reported from the step before they were eliminated from the model ( $p$ to remove $>0.05$ )

\begin{tabular}{|c|c|c|c|c|c|}
\hline Independent variables & $\begin{array}{l}\text { Order } \\
\text { eliminated }\end{array}$ & $\begin{array}{l}\text { Effect } \\
B\end{array}$ & $\begin{array}{l}\text { Standard } \\
\text { Error }\end{array}$ & $\begin{array}{l}\text { 95\% Confidence } \\
\text { Interval for } B\end{array}$ & $P$ \\
\hline Proportion of gender & 1 & 0.42 & 0.78 & $-1.21-2.05$ & 0.60 \\
\hline Mean age of users & 2 & -0.05 & 0.03 & $-1.1-0.01$ & 0.09 \\
\hline How often (seldom) & & 2.12 & 0.62 & $0.83-3.42$ & $<0.001$ \\
\hline Impact of design on the use & & 1.75 & 0.76 & $0.18-3.32$ & 0.031 \\
\hline
\end{tabular}


Figure 1: Difficulty hierarchy of everyday technologies as measured with META

(logits) Harder technology

\begin{tabular}{|c|c|c|}
\hline 1,38 & TV: video (with remote control) & $\mathrm{ICT}^{*}$ \\
\hline 0,96 & TV: DVD/ digital box (with remote control) & ICT \\
\hline 0,89 & Stereo: Portable CD & ICT \\
\hline 0,88 & Computer: print document & ICT \\
\hline 0,74 & Computer: write document & ICT \\
\hline 0,59 & Cellphone: Check missed call & $\mathrm{ICT}$ \\
\hline 0,58 & Cellphone: SMS/MMS & $\mathrm{ICT}$ \\
\hline 0,49 & Computer: find document & $\mathrm{ICT}$ \\
\hline 0,35 & Cell-phone: call & $\mathrm{ICT}$ \\
\hline 0,34 & Computer: e-mail & ICT \\
\hline 0,27 & Computer: search the internet & $\mathrm{ICT}$ \\
\hline 0,16 & Radio (with remote control) & $\mathrm{ICT}$ \\
\hline$-0,01$ & Computer: Internet banking & $\mathrm{ICT}$ \\
\hline$-0,08$ & Stereo (with remote control) & $\mathrm{ICT}$ \\
\hline$-0,08$ & Stereo: CD/LP(without remote control) & ICT \\
\hline$-0,18$ & Radio (without remote control) & $\mathrm{ICT}$ \\
\hline$-0,38$ & Dishwasher & $\mathrm{D}^{\dagger}$ \\
\hline$-0,58$ & Washing machine & $\mathrm{D}$ \\
\hline$-0,91$ & Coffeemaker & $\mathrm{D}$ \\
\hline$-0,91$ & Press-button telephone & $\mathrm{ICT}$ \\
\hline$-1,12$ & Micro & $\mathrm{D}$ \\
\hline$-1,27$ & TV: using remote control & $\mathrm{ICT}$ \\
\hline$-1,4$ & Electric kettle & $\mathrm{D}$ \\
\hline$-2,2$ & Stove & $\mathrm{D}$ \\
\hline
\end{tabular}

*ICT= Information and Communication Technology

${ }^{\dagger} \mathrm{D}=$ Domestic technology 